\title{
Subject Index Vol. 31, 1997
}

Acid solubility 212 Adolescents 161 Adsorption 434, 441 Aged 1

Aluminium 85 Amalgam restoration 19 Anemia 238, 244 Animal model 155 Antibacterial varnish 189, 361 Antibodies 35 Apatite 71

Artificial caries lesion 275 Aspartame 78 Atherosclerosis 180 Attitudes 91 Audit 97

Bactericidal effect 384 Bitewing radiography 19, 232 Brushite 71 Buffer capacity 148 Buffering 44

Calcium 429

fluoride 206

phosphate 434 Caries 78,141,201,238 -, deciduous 174

demineralization 125

diagnosis 232,329

prevalence 166

prevention 85 Cariostatic agents 244 Carious dentine 41,384 Cementum 390 Chewing gum 91,366 Children 166,180 Chlorhexidine 189,361 Chlorhexidine/xylitol 91 Class II amalgam restoration 24 Clinical trial 85,384 Clomipramine 155

Collagen 349 Colonization 349 Colony morphology 35 Computer-aided diagnosis 103 Coronal caries 405

Deep carious lesions 411 Defluoridation 390

Demineralization 103, 212, 216, 349,423, 429,457

Dental caries $1,8,13,103,148,155,161,194,244$

erosion 44

fluorosis 174

health survey 1

plaque 194,356,451 Dentifrice 418 Dentine 141,201,349 Dextranase inhibitor 441 DFS index 1

Diabetes mellitus, insulin-dependent 13

Diagnosis 97, 111

Diet 148

Dietary carbohydrates 180

fluoride supplements 174

nutrients 148

Digital Imaging Fiber-Optic Translllumina-

tion 103 Dose response 60 Dye 232

Egg yolk immunoglobulin 268 Elderly subjects 91,249 Electrical resistance 111 Electronic conductance 224 Enamel 60,71,212,216,418,429

defects 259

lesion 132

stain 91 Epidemiology 1 Erupting teeth 336

Fat intake 180

Fiber-optic transillumination 103, 232 
Field trial 329

Fissure caries 329

- $\quad$ morphology 336

Flow rate 44

Fluorapatite 397

Fluorescence 125

Fluoridated milk 212

Fluoride 30, 60, 132, 141, 148, 155,244, 259, 275, 356, 366, 373, 379, 390, 418, 457

analysis 451

concentrations 166

content 212

uptake 206

varnish 361 Food 429

fortification 238 Fractals 186

Germany 166 Glass ionomer 379

- cement 418

microelectrode $55 \alpha$-ט̂-Glucans 441 Glycolysis 373 Groove(s) 141

morphology 336 Growth 35

Hexetidine 457 Hidden caries, occurrence 30 Histology 336 Histopathology 224 Hydroxyapatite 35, 397 Hyposalivation 155

Incidence 8,249,405 Inorganic composition 356 In situ models 194 Interdental plaque 189,361

Intracellular $\mathrm{pH} 373$ Intraoral distribution 249

- $\quad$ model 60

Iodide penetrability 429 Ion-sensitive field effect transistor 55 Iron 238

- $\quad$ fortification 244

Lactobacilli 91 Laser 125,397

- $\quad$ fluorescence 132

Light microscopy 423

Logistic models 405

Microbiological sampling 41 Microbiology 411 Microhardness 275 Microradiography 125,423

Minimal demineralisation-inhibiting

concentration 457 Models 50 Monetite 71 Mouth rinse 268,418 Mutans streptococci 91, 189, 361,379

Non-linear dynamic 186 Nutritional status 259

Occlusal caries 97, 111,224, 336

- $\quad$ plaque 336

Occurrence 161

K A RG E R C 1997 S. KargeГ AG, Basel

E-Mail karger@karger.ch Fax + 41613061234 http://www. karger. ch

Older adults 405 Oral cleft 8

- $\quad$ health 180

Organic content 336

Passive immunization 268 Periodontitis $180 \mathrm{pH} 44$

cycling 216

- model 206 -, dental 50 Phosphate 429 Pilocarpine 155 Plaque 50,379

formation 194 
pH 55,366,451 Polarization 161 Polymers 216 Polysaccharides 216,356 Prenatal fluorides 174 Prevention 161, 180 Primary dentition 8 Proteolytic degradation 349 Proton excretion 373

Quantification 132

Race 1

Radiographic assessment 224

Radiography 24

Radiopaque and radiolucent dentine 19

Rat 155,390

Remineralization $132,216,418,423$

Remote effect 275

Resin composite 379

Resin-modified glass ionomer cement

275 Restorative materials $384 \operatorname{Root}(\mathrm{s}) 201$

caries 201,423

- index 249

remineralization 206 Rubber dam 41

Saliva 44, 189,366 -, flow rate 148

- $\quad$ substitutes 216

Salivary flow 91

Saturation 429

Secondary caries diagnosis 19, 24

Small volumes 55

Social status 259

Sodium hexafluorosilicate 206

Soft drinks 44

Solubility 71

Sorbitol 50

Stepwise excavation 411

Streptococci 373

Streptococcus mutans 268, 429

sobrinus 441

-, chain length 35 Sucrose 50, 78, 356, 429 Sugar 238, 244

System of expansion-contraction 186

Toothpaste 85, 141 Transillumination 103 Two-solution fluoride rinse 206

Ultrastructural feature 336

Varnishes 201 Visual assessment 224

Water fluoridation 166

Whitlockite 71

Wire oxide electrode 55

Xerostomia 91,216 Xylitol 91

Yeasts 13,91

Zinc 434

466

Caries Res Vol. 31, 1997

Subject Index 